\title{
Optical Wavelength Converters Based on Four Wave Mixing in SOA-MZI Configuration
}

\author{
Rajni ${ }^{1}$, Anu Sheetal \\ Department of Electronics and Communication, Guru Nanak Dev University (RC Gurdaspur)
}

\begin{abstract}
Wavelength converter plays an important role for increasing the capacity and flexibility of future broadcast network. This paper investigates the performance of a $10 \mathrm{~Gb} / \mathrm{s}$ the SOA based FWM wavelength converter. All performances are analyzed in terms of its shifted wavelength conversion efficiency, Q-factors and converted signal powers. The converters are modeled and simulated using Optisystem7 Software, by varying the probe signal wavelength and power. It was found that conversion efficiency and OSNR of the converted signal both decreased at large detuning wavelengths. Similarly, higher total SOA input powers worsened the conversion efficiency, but steadily improved the OSNR. FWM wavelength converter had the lowest conversion efficiency for CW sources.
\end{abstract}

\section{Introduction}

Optical wavelength converters are the key component for multi-wavelength optical transport networks and are provide wavelength conversion in optical domain without distortion of input signal [1]. The electro-optic converter is a straight forward solution for conversion. But electrooptic converter has limitations such as large power consumption and complexity. Wavelength conversions based on semiconductor optical amplifiers (SOAs) and semiconductor lasers have been focus on research interests during the last few years $[1,2]$.The capacity of wavelength division multiplexing (WDM) is usually limited by several factors, such as number of channels which can be deployed, wavelength congestion level and system management. A wavelength converter can be used to overcome such network limitations. A wavelength converter is an optical device which is used for the purpose of converting an injected signal of light from one wavelength to the desired wavelength in a system or network. Recently the four wave mixing technique occurring within a semiconductor optical amplifier has been one of the most favorable methods of wavelength conversion, offering benefits to the system designer [3].

FWM offers transparency to the bit rate and modulation format and hence preserves both the phase and amplitude information. FWM is the only method with transparent optical properties, due to unchanging nature of the optical properties of the information signal during the conversion process occurring within a SOA [4].Additionally, SOA-based FWM wavelength conversion offers many other attractions; for example high bit capability-up to $10 \mathrm{~Gb} / \mathrm{s}$ [5] or even $20 \mathrm{~Gb} / \mathrm{s}$ [6] have been demonstrated. FWM methods are also capable of operating at high bit rate capability without compromising the extinction ratio.

Wavelength conversion exploiting the SOA nonlinearities exhibits different advantages and disadvantages, depending on the operation. For example, the main advantages of XGM wavelength converters are their simplistic configurations, high conversion efficiencies and high bit rate capabilities.SOA based wavelength converters can also be arranged so as to possess polarization immunity, as well as wavelength insensitivity, towards the incoming input data [7].Polarization insensitivity is crucial in optical networks, as the polarization state in fibers can vary with environmental conditions.

\section{Basic operations}

\subsection{Four wave mixi}

In this, two optical waves with identical states of polarization are coupled into a saturated SOA. The pump signal (Epump) at a frequency of GDpump is typically stronger as compared to probe signal (Eprobe) at a frequency of GOprobe, which contains the data to be wavelength converted. The data signal is kept small, when compared to the pump, so as to avoid any unwanted gain modulation which can affect the performance of the converted signal during the conversion process [8].

\footnotetext{
${ }^{1}$ Corresponding author: rajni_kundan@yahoo.com
} 

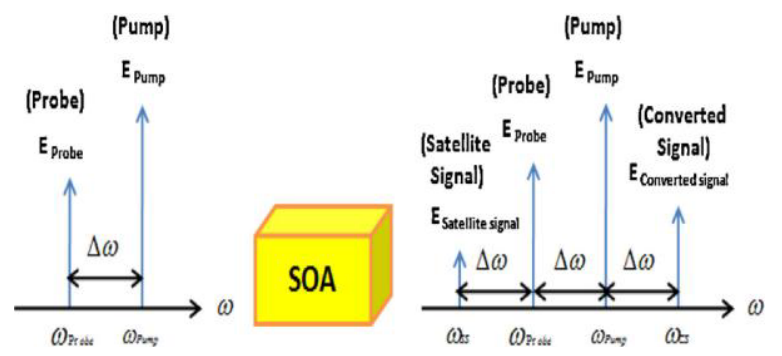

Figure 1. FWM scheme in SOA

Inside the active region of the SOA, the beatings of the two co-propagating waves modulates the carrier density, and therefore generates dynamic gain and index gratings. This non-linear interference produces new waves do not overlap with other wavelengths [11]. The intensity of these newly generated waves, known as the Econverted_signal and Esatellite_signal, is proportional to the product of the interacting wave intensities. Additionally, the phase and frequency of the newly generated waves is a linear combination of the wave present in the SOA active medium [12]. Eprobe is upconverted to a longer wavelength, Econverted_signal at an optical frequency of $\mathrm{Goss}=\mathrm{GOpump}+\Delta \mathrm{G}$, whereby $\Delta \mathrm{GO}$ refers to the detuning frequency. Econverted_signal is the phase conjugate replica of the original input signal, Eprobe. Increasing the gain, saturation power and carrier recovery rate of an SOA are a few practical methods of improving the conversion efficiency of a FWM wavelength converter [13].

$\eta=10 \log \frac{P_{\text {out }}\left(\lambda_{\text {converted_signal }}\right)}{P_{\text {in }}\left(\lambda_{\text {pump_signal }}\right)}$

Studies on FWM-based wavelength converters utilizing SOAs have been proposed in much previous works, due to numerous advantages offered by nonlinearities of SOA. For example D'Ottavi et al. [13] proved that use of a long SOA could improve the performance of the conversion at a data rate of $10 \mathrm{~Gb} / \mathrm{s}$. Also, Hsu et al. [14] claimed that higher number conversion efficiency and wider conversion range can be achieved by applying an assisted holding beam into the SOA. The assisted beam, which was used to saturate the SOA, can improve the conversion efficiency and signal to-background ratio. Additionally, unlike most of the proposed techniques using coherent light as the source, Forsyth et al. [15] demonstrated in novel work that SOA FWM can be obtained from an amplified spontaneous emission (ASE) source.

\section{Modeling and simulation}

The simulation was carried out at a central frequency of 193.5THz. A scheme of the modeling blocks was created, based on a CW laser source for the FWM-based wavelength converter, and is shown in Fig.2. The SOA parameters varied in this modeling. The pump and probe from the $\mathrm{CW}$ laser source was set to have SOA input powers of $5 \mathrm{dBm}, 3 \mathrm{dBm},-5 \mathrm{dBm}$ and $-3 \mathrm{dBm}$ respectively. The probe signal was modulated by a NRZ electrical driver, with a PRBS signal. The signal wavelength was varied in accordance with the desired detuning frequency. The pump and probe signals each passed through individual polarization controllers, PC1, $\mathrm{PC} 2, \mathrm{PC} 3$ and PC4, in order to match the state of polarization between them. This was a necessary condition to ensure efficient FWM occurred in SOA before being multiplexed by the multiplexer.

The multiplexed signals were then amplified by an EDFA, having a fixed gain of $10 \mathrm{~dB}$ and with an average noise figure of $4.2 \mathrm{~dB}$. The EDFA was used to increase the bandwidth optical band pass filter (BPF) was placed just after the EDFA so that any additional ASE outside the signal bandwidth was suppressed, hence increasing the OSNR of the converted signal. A nominal bias current of $250 \mathrm{~mA}$ was injected into the SOA as soon as the signals entered it, which then generated the converted signal through the FWM process. Another $0.4 \mathrm{~nm}$ Fabry Perot Optical filter, placed just after the SOA and centered on the converted signal wavelength, ensured clean capture of the converted wave with complete pump and original signal removal.

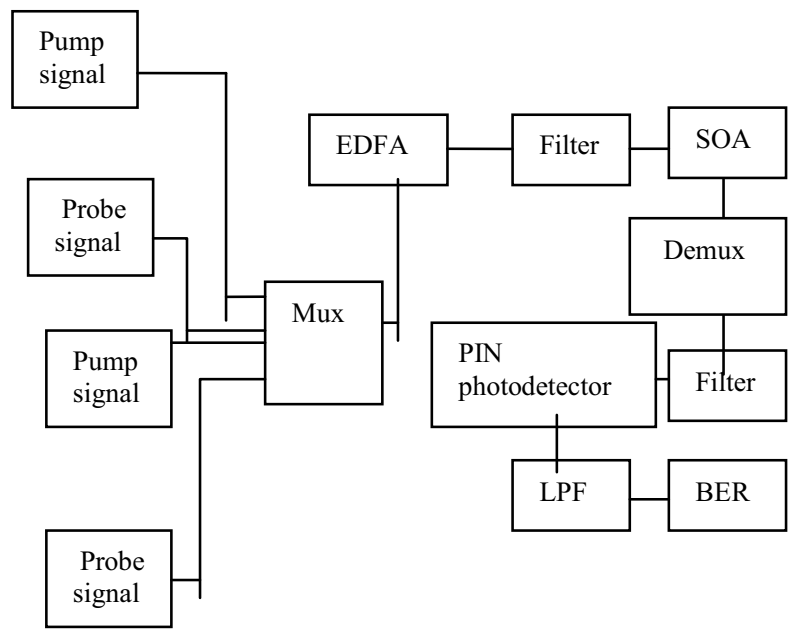

Figure2. Block diagram of 4 channels FWM wavelength converter.

\section{Results and discussions}

The simulation has been carried out for the set-up as shown in Fig.2. The converted signal power obtained as a function of wavelength shift. For showing the down conversion, an input with a frequency difference was taken. The pump signal was generated with the help of a $\mathrm{CW}$ laser at a wavelength of $1550 \mathrm{~nm}$ and the probe signal was also generated using a $\mathrm{CW}$ laser at a wavelength of $1552 \mathrm{~nm}$. For the purpose of up conversion, the pump and the probe signals are generated at $1550 \mathrm{~nm}$ and $1548 \mathrm{~nm}$ respectively. These are given as the co-polarized inputs into the SOA.

Effect of Bit Rate: The results were obtained for the bit rates of $10 \mathrm{~Gb} / \mathrm{s}$ respectively. 


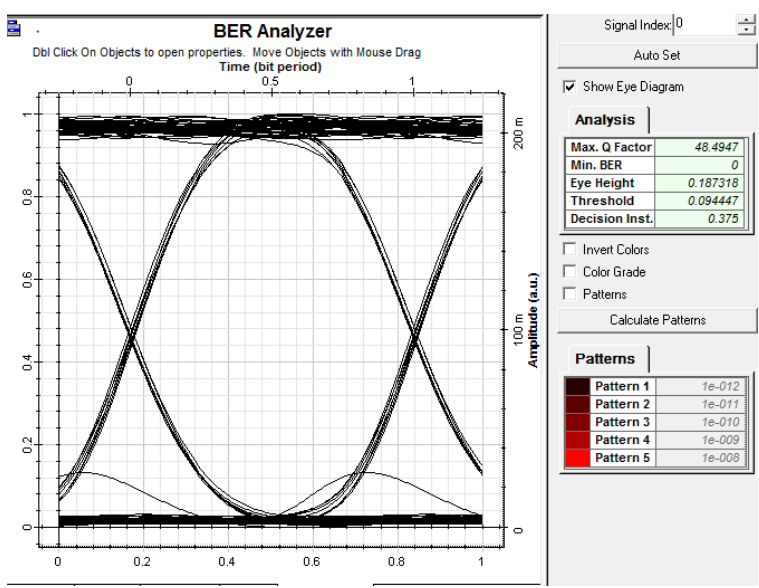

Figure 3. Eye diagram for signal received i.e. $193 \mathrm{THz}$

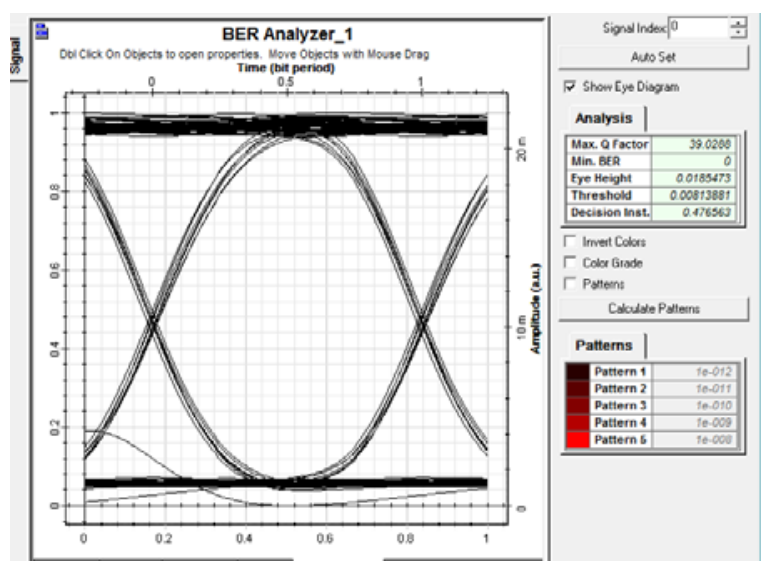

Figure 4. Eye diagram for $193.1 \mathrm{THz}$ signal

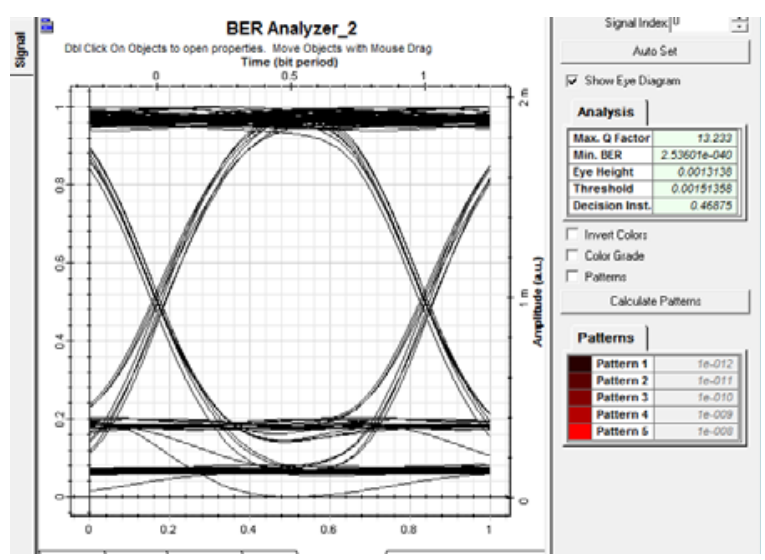

Figure 5. Eye diagram for 193.2THz signal

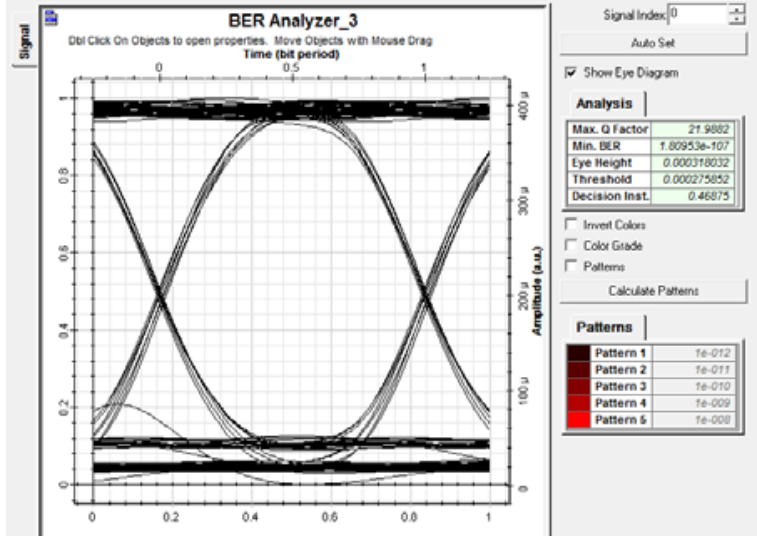

Figure 6. Eye diagram for $193.3 \mathrm{THz}$ signal

\section{Conclusion}

We have simulated and investigated various effects of the FWM wavelength conversion process occurring within a SOA medium, and have gathered useful data which could be used to evaluate the potential of such a scheme for applications in future, all-optical converter systems. The analysis was done through different channels at different frequency and power. The process occurred within an SOA medium which provides numerous advantages over fiber optic cable such as transparency to bit rates and modulation formats. This scheme of wavelength converter using FWM in SOA holds great importance for the future all optical converter system. In addition to this, we have also analyzed the various parameters of FWM in SOA which includes effect of bit rate and varied frequency and power.

\section{References}

1. M. Kovacevic, A. Acampora, Benefits of wavelength translation in all optical clear channel networks, IEEE J. Sel. Area Comm. 14 (1996) 69-79.

2. T. Durhuus, B. Mikkelson, C. Joergensen, S.L. Danielson, K.E. Stubkjaer, All optical wavelength conversion by semiconductor optical amplifiers, J. Lightwave Technol. 14 (1996) 942-953.

3. Surinder Singh, R.S. Kaler, All optical wavelength converters based on cross phase modulation in SOA-MZI configuration, Optik 118(2007)390-394.

4. D.D. Marcenac, D. Nesset, A.E. Kelly, D. Gavrilovic, $40 \mathrm{~Gb} / \mathrm{s}$ transmission over $103 \mathrm{~km}$ of NDSF using polarization independent mid-span spectral inversion by four-wave mixing in a semiconductor optical amplifier, Electron. Lett. 34(1) (1998) 100-101.

5. S. Singh, R.S. Kaler, $20-\mathrm{Gb} / \mathrm{s}$ and higher bit rate optical wavelength conversion for RZ-DPSK signal based on four-wave mixing in semiconductor optical amplifier, J. Fiber Integr. Optics 26 (5) (2006) 295-308. 
6. S. Singh, R.S. Kaler, Wide band optical wavelength converter based on four-wave mixing using optimized semiconductor optical amplifier, J. Fiber Integr. Optics 25 (3) (2007) 213-230.

7. D.I. Forsyth, M.J. Connelly, Dual semiconductor optical amplifier polarization independent wavelength conversion using four-wave mixing with optoelectronic feedback, in: Conference Proceedings - Lasers and Electro-Optics Society Annual Meeting, vol. 1, 2004, pp. 396-397.

8. Farah Diana Mahad, Abu Sahmah M. Supa'at, Sevia Mahdaliza Idrus, David Forsyth, Comparative performance testing of SOA wavelength conversion techniques for future alloptical systems, Optik 124 (2013) 1254- 1259.

9. K. Obermann, S. Kindt, D. Breuer, K. Petermann, Performance analysis of wavelength converters based on cross-gain modulation in semiconductor-optical amplifiers, J. Lightwave Technol. 16 (1) (1998) 78-85.

10. S. Wu, X. Sun, M. Zhang, Characteristics of the wavelength converted signal in wavelength conversion based on cross-gain modulation, Int. J. Infrared Millimeter Waves 23 (2) (2002) 309316.

11. Farah Diana Mahad, Abu Sahmah M. Supa'at, Sevia Mahdaliza Idrus, David Forsyth, Analyses of semiconductor optical amplifier (SOA) fourwave mixing (FWM) for future all-optical wavelength conversion, Optik 124 (2013) 1-3.

12. M.Y. Jamroa, J.M. Seniora, M.S. Leesonb, G. Murtaza, Chirp in a wavelength converter based on a symmetrical-MZI employing SOAs, Opt. Commun. 209 (4-6) (2002) 321-328.

13. A. D’Ottavi, P. Spano, G. Hunziker, R. Paiella, R. Dall'Ara, G. Guekos, K.J. Vahala, Wavelength conversion at $10 \mathrm{~Gb} / \mathrm{s}$ by four-wave mixing over a 30-nm interval, IEEE Photon. Technol. Lett. 10 (7) (1998) 952-954.

14. D. Hsu, S. Lee, P. Gong, Y. Lin, S.S.W. Lee, M.C. Yuang, High efficiency wide-band SOAbased wavelength converters by using dualpumped four-wave mixing and an assist beam, IEEE Photon. Technol. Lett. 16 (8) (2004) 1903-1905.

15. D.I. Forsyth, M.J. Connelly, Spectrum-sliced wavelength conversion using four wave mixing from a semiconductor optical amplifier, in: Optical Amplifiers and Their Applications, Technical Digest (CD) (Optical Society of America, 2005), Paper TuC3,( 2005). 